\title{
DNA Damage Repair and Drug Efflux as Potential Targets for Reversing Low or Intermediate Ciprofloxacin Resistance in E. coli K-12
}

\author{
Rasmus N. Klitgaard ${ }^{1}$, Bimal Jana ${ }^{2}$, Luca Guardabassi², Karen L. Nielsen ${ }^{3}$ and \\ Anders Løbner-Olesen ${ }^{1 *}$
}

${ }^{1}$ Department of Biology, Section for Functional Genomics, University of Copenhagen, Copenhagen, Denmark, ${ }^{2}$ Department of Veterinary and Animal Sciences, Section for Veterinary Clinical Microbiology, University of Copenhagen, Copenhagen, Denmark, ${ }^{3}$ Department of Clinical Microbiology, Center for Diagnostics, Rigshospitalet, Copenhagen, Denmark

\section{OPEN ACCESS}

Edited by:

Sanna Sillankorva,

University of Minho, Portugal

Reviewed by:

César de la Fuente,

Massachusetts Institute

of Technology, United States

Munawar Sultana,

University of Dhaka, Bangladesh

Azucena Mora Gutiérrez,

Universidade de Santiago

de Compostela, Spain

Catherine M. Logue,

University of Georgia, United States

${ }^{*}$ Correspondence:

Anders Løbner-Olesen lobner@bio.ku.dk

Specialty section:

This article was submitted to Antimicrobials, Resistance

and Chemotherapy,

a section of the journal

Frontiers in Microbiology

Received: 15 March 2018 Accepted: 11 June 2018

Published: 02 July 2018

Citation:

Klitgaard RN, Jana B, Guardabassi L,

Nielsen $K L$ and Løbner-Olesen $A$ (2018) DNA Damage Repair and Drug

Efflux as Potential Targets for Reversing Low or Intermediate Ciprofloxacin Resistance in E. coll K-12. Front. Microbiol. 9:1438. doi: 10.3389/fmicb.2018.01438
Ciprofloxacin is a potent antibacterial drug that is widely used in human clinical applications. As a consequence of its extensive use, resistance has emerged in almost all clinically relevant bacterial species. A mean to combat the observed ciprofloxacin resistance is by reversing it via co-administration of a potentiating compound, also known as a helper drug. Here, we report on the current advances in identifying ciprofloxacin helper drugs, and put them into perspective of our own findings. We searched for potential helper drug targets in Escherichia coli strains with different levels of ciprofloxacin resistance using transcriptomics i.e., RNAseq and by deletion of genes associated with hyper-susceptibility to ciprofloxacin. Differential gene expression analysis of the highly ciprofloxacin resistant uropathogenic E. coli strain, ST131 UR40, treated with a clinically relevant concentration of ciprofloxacin $(2 \mu \mathrm{g} / \mathrm{mL})$, showed that the transcriptome was unaffected. Conversely, genetic screening of 23 single gene deletions in the high-level ciprofloxacin resistant laboratory derived E. coli strain, LM693, led to a significant decrease in the minimal inhibitory concentration for several genes, including genes encoding the AcrAB-ToIC efflux pump, SOS-response proteins and the global regulator Fis. In addition, deletion of acrA, to/C, recA, or rec $C$ rendered two $E$. coll strains with intermediate susceptibility to ciprofloxacin fully susceptible according to the CLSI recommended breakpoint. Our results corroborate the AcrAB-TolC efflux pump and the SOS response proteins, RecA and RecC, as potential targets for ciprofloxacin helper drugs in treatment of human bacterial infections caused by E. coli strains with intermediate sensitivity to ciprofloxacin.

Keywords: antibiotic resistance, ciprofloxacin, helper drugs, RNA-Seq, transcriptomics

\section{INTRODUCTION}

Fluoroquinolones are some of the most prescribed antibacterial drugs in the world, commonly used for the treatment of urinary tract infections and sinusitis (Emmerson and Jones, 2003; Linder et al., 2005; Mitscher, 2005), but this has not always been the case. For the first two decades after the discovery of nalidixic acid in 1962, and its introduction into the clinic in 1964, the quinolones

Abbreviations: MIC, minimal inhibitory concentration; ST, sequence type. 
were only used to treat uncomplicated urinary tract infections. This changed with the release of the second generation quinolones, including ciprofloxacin, which showed significant activity outside the urinary tract and against a broad spectrum of both Gram-negative and Gram-positive bacteria. Ciprofloxacin acts by binding to its targets, DNA gyrase and topoisomerase IV, inhibiting the native ability of these two enzymes to re-ligate double stranded DNA breaks, in turn leading to fragmentation of the chromosome. Due to its mechanism of action it is sometimes referred to as topoisomerase poison (Aldred et al., 2014). Inevitably, considering its extensive use and misuse, resistance toward ciprofloxacin has increased in almost all clinically relevant bacteria (Werner et al., 2011; Dalhoff, 2012). One method to overcome antibacterial resistance is by combinatorial treatment with a potentiating compound, also known as a helper drug. A helper drug is by definition non-antibacterial when administered alone, but it enhances the activity of the antibiotic when used in concert. The potentiating effect of a helper drug can be achieved by either direct inhibition of the resistance mechanism or by targeting endogenous cellular components and pathways like, cell membranes, efflux pumps and cellular repair systems. A classic example of targeting the resistance mechanism is the combination of amoxicillin and the $\beta$-lactamase inhibitor clavulanic acid (White et al., 2004). Highlevel ciprofloxacin resistance is primarily associated with multiple target site mutations in gyrA and parC, encoding subunits of the DNA gyrase and topoisomerase IV, respectively (Aldred et al., 2014). Since 1998 three different plasmid-mediated ciprofloxacin resistance mechanisms have been identified; (i) target protection (Qnr proteins), (ii) efflux pumps (QepA and OqxAB) and (iii) drug modification (AAC $\left(6^{\prime}\right)$-Ib-cr acetyltransferase) (RodríguezMartínez et al., 2016).

\section{Potential Ciprofloxacin Helper Drug Targets}

Studies of the endogenous cellular mechanisms involved in ciprofloxacin susceptibility and resistance evolution have revealed more than two dozen gene deletions that lead to increased ciprofloxacin susceptibility in wild-type Escherichia coli (Cirz et al., 2005; Tamae et al., 2008; Liu et al., 2010; Yamada et al., 2010). Thus, suggesting the gene products as potential ciprofloxacin helper drug targets. Recently, Tran et al. identified 23 single gene deletions that increased the ciprofloxacin susceptibility of a laboratory derived resistant E. coli strain. The most significant increase in ciprofloxacin susceptibility was observed for the deletion of SOS-response genes directly involved in DNA damage repair, and the genes encoding the AcrAB-TolC efflux pump (Tran et al., 2016). Recacha et al. recently showed that deletion of $\operatorname{rec} A$ rendered a laboratory derived E. coli strain with intermediate sensitivity to ciprofloxacin clinically susceptible in vitro. In addition, the in vivo efficacy of ciprofloxacin against the same strain was significantly increased in a peritoneal sepsis murine model (Recacha et al., 2017). Thus, the current evidence suggests that targeting the repair of ciprofloxacin induced DNA damage or the efflux pump AcrABTolC are the most promising strategies for ciprofloxacin helper drugs. Here, we used a combined transcriptomic and genetic approach in an attempt to both identify novel helper drug targets, as well as further assess the potential of known helper drug targets in laboratory derived E. coli strains with different levels and mechanisms of ciprofloxacin resistance.

\section{MATERIALS AND METHODS}

\section{Bacterial Strains and Plasmids}

Strains LM693 and LM862 were obtained from Diarmaid Hughes from Uppsala University. LM693 is isogenic to the commonly used laboratory strain, MG1655, besides two gyrA mutations, S83L and $\mathrm{D} 87 \mathrm{~N}$, and one parC mutation, S80I. LM862 is also isogenic to MG1655, but with one gyrA S83L mutation and one parC S80I mutation. ST131 UR40 has two gyrA mutations, S83L and D87, and two parC mutations, S80I and E84V, and carries aac-6 $-\mathrm{Ib}$-cr on a plasmid (Cerquetti et al., 2010). The aac-6'-Ib-cr carrying plasmid pRNK1 (was constructed as follows: $a a c-6^{\prime}-I b-c r$ gene was amplified by PCR from ST131 UR40, using the following primers: GATCGGATCCATGAGCAACGCAAAAACAAAGTT AGGC and CATCGAATTCTTAGGCATCACTGCGTGTTCGC, and cloned into pMW119 (Nippon Gene, Toyama, Japan) using BamHI and EcoRI. The qnrS-carrying plasmid pRNK9 was constructed as follows: $q n r S$ was amplified by PCR from the clinical $E$. coli isolate EC38 using the following primers: GATCGGATCCATGGAAACCTACAATCATACATAT CGGC and GATCAAGCTTTTAGTCAGGATAAACAACAAT ACCCAGTGC, and cloned into pMG25 using BamHI and HindIII (M. Mikkelsen and K. Gerdes, unpublished). pRNK1 (4796 bp) and pRNK9 (4723 bp) was then introduced in LM862 by electroporation. Strain EC38 was isolated from a patient with a urinary tract infection at Hvidovre Hospital, Denmark.

\section{Genetic Screening and MIC Tests}

For the genetic screen, P1 phage lysates were prepared from the relevant Keio collection strains (Baba et al., 2006) and used for transduction into LM693 and LM862. All the transduced strains were verified by PCR. The ciprofloxacin MICs for LM693 and derived strains were determined using E-tests $(0.002-32 \mu \mathrm{g} / \mathrm{ml}$, BioMerieux) and according to the manufacturer's guidelines. The MICs for LM862 and derived strains were determined by broth micro-dilution using cation adjusted Mueller Hinton broth II with $1 \mathrm{mM}$ and $10 \mu \mathrm{M}$ IPTG for pRNK1 and pRNK9, respectively. The two different IPTG concentrations were used to obtain a ciprofloxacin MIC for LM862/pRNK1 and LM862/pRNK9 of $2 \mu \mathrm{g} / \mathrm{mL}$ i.e., within the CLSI intermediate susceptible range. The reference E. coli strain ATCC 25922 was used as standard in all MIC tests and the susceptibility was evaluated according to CLSI recommended breakpoints.

\section{Checkerboard Assay}

All wells in a micro-titter plate were filled with $100 \mu \mathrm{l}$ cation adjusted Mueller Hinton broth II $(200 \mu \mathrm{L}$ in the negative control wells). Copper phtalocyanine- $3,4^{\prime}, 4^{\prime \prime}, 4^{\prime \prime \prime}$-tetrasulfonic acid, was added to the first row, followed by serial dilution along the 
abscissa, leading to a start concentration of $100 \mu \mathrm{M}$. Hereafter ciprofloxacin was serial diluted along the ordinate, giving a start concentration of 2 and $64 \mu \mathrm{g} / \mathrm{ml}$ for LM862 and LM693, respectively. Hundred microliter diluted culture with an OD600 of 0.001 was then inoculated in each well and the plates were incubated at $37^{\circ} \mathrm{C}$ for $24 \mathrm{~h}$.

\section{RNA-Sequencing}

Ciprofloxacin was added to a culture of ST131 UR40, which had been growing exponentially for more than six generations, to a final concentration of $2 \mu \mathrm{g} / \mathrm{ml}$. Samples for RNA isolation were taken at $0 \mathrm{~min}$ (prior to ciprofloxacin addition) and 30 and $90 \mathrm{~min}$ after ciprofloxacin addition, which has previously been shown to be long enough to induce fragmentation of the E. coli chromosome (Charbon et al., 2014). Total RNA was isolated using a Thermo Scientific GeneJET RNA isolation kit. Dnase treated with TURBO DNA-free kit from Ambion. rRNA was depleted using an Illumina Ribo-zero rRNA removal kit, followed by RNA-Seq library prep using an Illumina TruSeq Stranded mRNA Library Prep Kit. Sequencing was performed on an Illumina Miseq with a Miseq reagent kit v3. (75 bp pairedend) from Illumina. Data analysis was performed in Rockhopper ver.2.03 (McClure et al., 2013). E. coli NA114 (ST131) (accession number: NC_017644) was used as reference genome (Avasthi et al., 2011). The percentage of successfully aligned reads varied from 91 to $88 \%$ of the total read count. The sequencing data files and the Rockhopper results from the differential gene expression analysis are available from the Gene Expression Omnibus (GEO: GSE89507).

\section{RESULTS}

\section{Identification of Helper Drug Targets by Genetic Screening}

As mentioned in the introduction several single gene deletions are known to increase the ciprofloxacin susceptibility of E. coli. To further assess the helper drug target potential of these genes, 23 single gene deletions were introduced into the highlevel ciprofloxacin resistant E. coli strain LM693 (MIC of 24$32 \mu \mathrm{g} / \mathrm{ml}$ ) (Marcusson et al., 2009) and tested for hypersusceptibility toward ciprofloxacin (Table 1). LM693 is isogenic to the commonly used laboratory strain MG1655 besides two GyrA mutations; S83L and D87, and one ParC mutation; S80I. Even though nine of the mutant strains showed a three to four fold reduction in the MIC, none of them were found to be susceptible according to the CLSI MIC breakpoint for ciprofloxacin $(\leq 1 \mu \mathrm{g} / \mathrm{mL})$. Our results therefore indicate that none of the tested gene-knockouts identify valid helper drug targets in high-level ciprofloxacin resistant $E$. coli strains. However, they could potentially be used as helper drug targets in bacteria with intermediate susceptibility to ciprofloxacin. To create two strains with intermediate ciprofloxacin susceptibility, we constructed the plasmids pRNK1 and pRNK9 carrying the ciprofloxacin resistance determinants aac- $6^{\prime}-\mathrm{Ib}-\mathrm{cr}$ and qnrS, respectively. AAC- $6^{\prime}-\mathrm{Ib}-\mathrm{cr}$ inactivates ciprofloxacin by $\mathrm{N}$-acetylation of the amino nitrogen of its piperazinyl substituent
(Robicsek et al., 2006), while QnrS acts as a DNA mimic, binding to and protecting the gyrase from the action of ciprofloxacin (Rodríguez-Martínez et al., 2016). Introduction of pRNK1 and pRNK9 into strain LM862, which carries GyrA S83L and ParC S80I mutations, increased the MIC from 1 to $2 \mu \mathrm{g} / \mathrm{ml}$, i.e., into the CLSI intermediate susceptible MIC range. We then evaluated the ability of seven of the most promising gene deletions described above to reduce the ciprofloxacin MIC of LM862/pRNK1 and LM862/pRNK9. Four of the gene deletions (acrA, tolC, recA, and $r e c C$ ) rendered both strains susceptible to ciprofloxacin (Table 2). To assess whether inhibition of RecA was an amenable strategy for potentiation of ciprofloxacin, synergy between ciprofloxacin and a RecA inhibitor, copper phtalocyanine-3,4 $4^{\prime} 4^{\prime \prime}, 4^{\prime \prime \prime}$-tetrasulfonic acid (Alam et al., 2016), was tested by a checkerboard assay. However, we did not observe a reduction in the ciprofloxacin MICs for either LM693 or LM862.

\section{Identification of Helper Drug Targets by RNA Sequencing}

The E. coli clonal group ST131 has become the predominant E. coli lineage isolated from human extra-intestinal infections and is currently regarded a global problem in hospitals and clinical practices (Nicolas-Chanoine et al., 2014). Two independent studies have shown that more than $90 \%$ of ESBL-producing

TABLE 1 | MIC values for the single gene deletions in LM693.

\begin{tabular}{lc}
\hline Strain/single deletions & MIC $(\boldsymbol{\mu} \mathbf{g} / \mathbf{m l})$ \\
\hline LM693 & $24-32$ \\
tolC & 1.5 \\
acrA, acrB and fis & 2 \\
recC, xseA, xseB, uvrD, and $r e c A$ & 4 \\
ruvC and $d k s A$ & 6 \\
recG and $h / p A$ & 8 \\
pgm, $y b g F$ and $y b g C$ & 12 \\
deoR, $y d c S, y c i T$ and $y b j Q$ & 16 \\
$y g c O$ and $n l p C$ & 24 \\
rimK & $24-32$ \\
\hline
\end{tabular}

TABLE 2 | MIC values for the single gene deletions in LM862/pRNK1 and LM862/pRNK9.

\begin{tabular}{lcc}
\hline Strain & \multicolumn{2}{c}{ MIC $(\boldsymbol{\mu g} / \mathbf{m l})$} \\
\hline LM862 & 1 & 1 \\
LM862/Empty vectors & 1 & pRNK9 \\
& pRNK1 & 2 \\
LM862 & 2 & 0.5 \\
tolC & 0.25 & 0.5 \\
acrA & 0.25 & 0.5 \\
recA & 0.5 & 0.5 \\
recC & 0.5 & 1 \\
uvrD & 2 & 1 \\
xseA & 1 & 4 \\
fis & 2 &
\end{tabular}


ST131 isolates are also resistant to ciprofloxacin (Brisse et al., 2012; López-Cerero et al., 2014). Strain ST131 UR40 is resistant to high levels of ciprofloxacin due to GyrA mutations S83L and D87, and ParC mutations S80I and E84V (Cerquetti et al., 2010). Here we used RNA-Seq to map the transcriptomic changes during treatment of ST131 UR40 with a clinically relevant concentration of ciprofloxacin, $2 \mu \mathrm{g} / \mathrm{ml}$, which is approximately equal to the maximum serum concentration following oral administration of $500 \mathrm{mg}$ ciprofloxacin according to the FDA. The rationale behind this was to identify potential helper drug target genes that were upregulated upon ciprofloxacin exposure and thereby potentially involved in ciprofloxacin resistance. In contrast to the genetic screen, the RNA-Seq analysis would also reveal targets encoded by essential genes and non-coding RNA. The transcriptomic analysis did not show any non-ribosomal transcripts to be significantly upregulated in the presence of ciprofloxacin, i.e., with a false discovery rate of $<1 \%$ and more than two-fold expression change.

\section{DISCUSSION}

By utilizing a combination of "direct genetic screening" and differential gene expression analysis, we have attempted to identify potential genes suitable as targets for ciprofloxacin potentiating compounds. We did not find any genes to be significantly upregulated by ciprofloxacin, indicating that the transcriptome of ST131 UR40 was relatively unaffected by treatment with a sub-inhibitory and yet clinically relevant concentration of ciprofloxacin. The lack of an upregulation of the SOS response genes in the transcriptomic analysis suggests that the ciprofloxacin exposure did not cause sufficient DNA damage to induce a SOS response; hence it was not necessary for ST131 UR40 to upregulate any specific genes to cope with the presence of ciprofloxacin at a sub-inhibitory concentration.

The screening of selected mutant strains revealed a number of genes, which when deleted, lowered the MIC for ciprofloxacin significantly in LM693. These findings are in accordance with genes reported to contribute to high-level ciprofloxacin resistance by Tran et al. (2016). Treatment of bacteria with ciprofloxacin generates double stranded breaks in the DNA of the organism (Drlica et al., 2008), which in turn activates the SOS response. Seven of the tested gene deletions; $\operatorname{rec} A, \operatorname{rec} C, \operatorname{rec} G, u v r D$, $x s e A B$, and $r u v C$, which all significantly reduced the MIC of LM693, are part of the SOS response and involved in DNA damage repair (Chase and Richardson, 1974; Kuzminov, 1993; Michel, 2005). Thus, deletion of any of these seven genes likely lowers the ability of the bacteria to cope with ciprofloxacin induced DNA damage. Deletion of genes encoding the AcrAB-TolC efflux pump, or the global regulator Fis (Factor for inversion stimulation) showed the largest decreases in MIC values for LM693. The Fis protein has been shown to repress the gyrA and $g y r B$ promoters, thereby reducing the expression of the DNA gyrase (Schneider et al., 1999). Thus, deletion of fis likely increases DNA gyrase expression and the number of ciprofloxacin targets. As ciprofloxacin works as a topoisomerase poison, an increase in ciprofloxacin bound
DNA gyrase could potentially lead to an increase in double stranded breaks, explaining the decrease in MIC for the fis deletion strain. The fis deletion did not have the same effect on the intermediate susceptible strains LM862/pRNK1 and LM862/pRNK9, which may be explained by the relatively higher affinity of ciprofloxacin for its target in LM862, compared to that of LM693. Thus, the increase in expression of the DNA gyrase might lead to an increase in ciprofloxacin-gyrase complexes, but if the ciprofloxacin induced DNA damage is already at a level, where the DNA repair mechanisms cannot keep up, the fis deletion does not have a dramatic effect on the MIC.

Individual deletions of $a c r A, a c r B$, or tolC genes encoding the AcrAB-TolC efflux pump had a large effect on the ciprofloxacin susceptibility of both LM693 and LM862 strains. This was not surprising as overexpression of the AcrAB-TolC efflux system has been connected to ciprofloxacin resistance numerous times (Mazzariol et al., 2000). The deletion of acrA or tolC in the LM862 strains lowered the MIC beneath the CLSI susceptible breakpoint indicating that AcrAB-TolC efflux system is a potential target for ciprofloxacin potentiating compounds in intermediate susceptible E. coli. A number of AcrAB-TolC inhibitors have been identified (Chevalier et al., 2004; Bohnert et al., 2013; Aparna et al., 2014; Opperman et al., 2014; Yilmaz et al., 2015), two of which have been shown to decrease the MIC of ciprofloxacin in susceptible E. coli strains (Opperman et al., 2014; Yilmaz et al., 2015), but none of them are used in clinical practice so far.

Inhibition of RecA and thereby of the SOS response has been proposed as a strategy to fight antibiotic resistance numerous times (Blázquez et al., 2012; Culyba et al., 2015; Alam et al., 2016). Our finding, that deletion of $r e c A$ render intermediate susceptible strains of E. coli fully susceptible to ciprofloxacin is in accordance with recent observations by Recacha et al. (2017). Overall, this indicates that RecA could be a potential ciprofloxacin helper drug target.

Even though AcrAB-TolC or RecA deficiency rendered LM862/pRNK1 and LM862/pRNK9 susceptible to ciprofloxacin, the respective MICs were only two to four-folds lower than the susceptible MIC breakpoint. It therefore seems reasonable to assume that a given inhibitor should completely block the activity of either RecA or AcrAB-TolC in order for it to be an efficient helper drug. This hypothesis is backed by the failure of lowering the ciprofloxacin MIC of LM862 and LM693 with the RecA inhibitor phtalocyanine- $3,4^{\prime}, 4^{\prime \prime}, 4^{\prime \prime \prime}$-tetrasulfonic acid.

\section{CONCLUSION}

The findings of this study and the evidence given in the literature, indicates that reversal of ciprofloxacin resistance in high-level resistant $E$. coli strains by the use of helper drugs does not appear to be plausible. Conversely, targeting RecA, RecC or the AcrAB-TolC efflux pump is a likely feasible strategy for reversing ciprofloxacin resistance in $E$. coli strains with intermediate susceptibility to ciprofloxacin. However, it should be noted that there is a discrepancy between the MIC breakpoint 
for ciprofloxacin susceptibility proposed by the CLSI $(\leq 1 \mu \mathrm{g} / \mathrm{mL})$ and the EUCAST $(\leq 0.25 \mu \mathrm{g} / \mathrm{mL})$. Therefore, further in vivo studies are needed to asses if targeting either $\mathrm{RecA}$, $\mathrm{RecC}$, or AcrAB-TolC leads to a significant increase in the efficacy of ciprofloxacin against an intermediate susceptible $E$. coli strain.

\section{AUTHOR CONTRIBUTIONS}

RK carried out all experimental work, designed the study, analyzed the data, and prepared the final manuscript. AL-O supervised all aspects of the study and helped prepare the final manuscript. BJ assisted and supervised the experimental part

\section{REFERENCES}

Alam, M. K., Alhhazmi, A., Decoteau, J. F., Luo, Y., and Geyer, C. R. (2016). RecA inhibitors potentiate antibiotic activity and block evolution of antibiotic resistance. Cell Chem. Biol. 23, 381-391. doi: 10.1016/j.chembiol.2016.02.010

Aldred, K. J., Kerns, R. J., and Osheroff, N. (2014). Mechanism of quinolone action and resistance. Biochemistry 53, 1565-1574. doi: 10.1021/bi5000564

Aparna, V., Dineshkumar, K., Mohanalakshmi, N., Velmurugan, D., and Hopper, W. (2014). Identification of natural compound inhibitors for multidrug efflux pumps of Escherichia coli and Pseudomonas aeruginosa using in silico high-throughput virtual screening and in vitro validation. PLoS One 9:e101840. doi: 10.1371/journal.pone.0101840

Avasthi, T. S., Kumar, N., Baddam, R., Hussain, A., Nandanwar, N., Jadhav, S., et al. (2011). Genome of Multidrug-resistant uropathogenic Escherichia coli strain NA114 from India. J. Bacteriol. 193, 4272-4273. doi: 10.1128/JB.05413-11

Baba, T., Ara, T., Hasegawa, M., Takai, Y., Okumura, Y., Baba, M., et al. (2006). Construction of Escherichia coli K-12 in-frame, single-gene knockout mutants: the Keio collection. Mol. Syst. Biol. 2:msb4100050. doi: 10.1038/msb4100050

Blázquez, J., Couce, A., Rodríguez-Beltrán, J., and Rodríguez-Rojas, A. (2012). Antimicrobials as promoters of genetic variation. Curr. Opin. Microbiol. 15, 561-569. doi: 10.1016/j.mib.2012.07.007

Bohnert, J. A., Schuster, S., and Kern, W. V. (2013). Pimozide inhibits the AcrABTolC efflux pump in Escherichia coli. Open Microbiol. J. 7, 83-86. doi: 10.2174/ 1874285801307010083

Brisse, S., Diancourt, L., Laouénan, C., Vigan, M., Caro, V., Arlet, G., et al. (2012). Phylogenetic distribution of CTX-M- and non-extended-spectrum$\beta$-lactamase-producing Escherichia coli isolates: group B2 isolates, except clone ST131, rarely produce CTX-M enzymes. J. Clin. Microbiol. 50, 2974-2981. doi: 10.1128/JCM.00919-12

Cerquetti, M., Giufrè, M., García-Fernández, A., Accogli, M., Fortini, D., Luzzi, I., et al. (2010). Ciprofloxacin-resistant, CTX-M-15-producing Escherichia coli ST131 clone in extraintestinal infections in Italy. Clin. Microbiol. Infect. 16, 1555-1558. doi: 10.1111/j.1469-0691.2010.03162.x

Charbon, G., Bjørn, L., Mendoza-Chamizo, B., Frimodt-Møller, J., and Løbner-Olesen, A. (2014). Oxidative DNA damage is instrumental in hyperreplication stress-induced inviability of Escherichia coli. Nucleic Acids Res. 42, 13228-13241. doi: 10.1093/nar/gku1149

Chase, J. W., and Richardson, C. C. (1974). Exonuclease VII of Escherichia coli: mechanism of action. J. Biol. Chem. 249, 4553-4561.

Chevalier, J., Bredin, J., Mahamoud, A., Malléa, M., Barbe, J., and Pagès, J.-M. (2004). Inhibitors of antibiotic efflux in resistant Enterobacter aerogenes and Klebsiella pneumoniae strains. Antimicrob. Agents Chemother. 48, 1043-1046. doi: 10.1128/AAC.48.3.1043-1046.2004

Cirz, R. T., Chin, J. K., Andes, D. R., De Crécy-Lagard, V., Craig, W. A., and Romesberg, F. E. (2005). Inhibition of mutation and combating the evolution of antibiotic resistance. PLoS Biol. 3:e176. doi: 10.1371/journal.pbio.003 0176

Culyba, M. J., Mo, C. Y., and Kohli, R. M. (2015). Targets for combating the evolution of acquired antibiotic resistance. Biochemistry 54, 3573-3582. doi: 10.1021/acs.biochem.5b00109 of the RNA-seq. LG supervised and delivered the ST131 UR40 strain. KN performed genomic analyses and delivered the EC38 strain carrying the qnrS gene. All authors read and approved the final manuscript.

\section{FUNDING}

We acknowledge the financial support from the University of Copenhagen Centre for Control of Antibiotic Resistance (UCCare) and by the Center for Bacterial Stress Response and Persistence (BASP) funded by a grant from the Danish National Research Foundation (DNRF120).

Dalhoff, A. (2012). Global fluoroquinolone resistance epidemiology and implictions for clinical use. Interdiscip. Perspect. Infect. Dis. 2012:976273. doi: $10.1155 / 2012 / 976273$

Drlica, K., Malik, M., Kerns, R. J., and Zhao, X. (2008). Quinolone-mediated bacterial death. Antimicrob. Agents Chemother. 52, 385-392. doi: 10.1128/AAC. 01617-06

Emmerson, A. M., and Jones, A. M. (2003). The quinolones: decades of development and use. J. Antimicrob. Chemother. 51(Suppl. 1), 13-20. doi: $10.1093 / \mathrm{jac} / \mathrm{dkg} 208$

Kuzminov, A. (1993). RuvA, RuvB and RuvC proteins: cleaning-up after recombinational repairs in E. coli. Bioessays 15, 355-358. doi: 10.1002/bies. 950150511

Linder, J. A., Huang, E. S., Steinman, M. A., Gonzales, R., and Stafford, R. S. (2005). Fluoroquinolone prescribing in the United States: 1995 to 2002. Am. J. Med. 118, 259-268. doi: 10.1016/j.amjmed.2004.09.015

Liu, A., Tran, L., Becket, E., Lee, K., Chinn, L., Park, E., et al. (2010). Antibiotic sensitivity profiles determined with an Escherichia coli gene knockout collection: generating an antibiotic bar code. Antimicrob. Agents Chemother. 54, 1393-1403. doi: 10.1128/AAC.00906-09

López-Cerero, L., Navarro, M. D., Bellido, M., Martín-Peña, A., Viñas, L., Cisneros, J. M., et al. (2014). Escherichia coli belonging to the worldwide emerging epidemic clonal group O25b/ST131: risk factors and clinical implications. J. Antimicrob. Chemother. 69, 809-814. doi: 10.1093/jac/dkt405

Marcusson, L. L., Frimodt-Møller, N., and Hughes, D. (2009). Interplay in the selection of fluoroquinolone resistance and bacterial fitness. PLoS Pathog. 5:e1000541. doi: 10.1371/journal.ppat.1000541

Mazzariol, A., Tokue, Y., Kanegawa, T. M., Cornaglia, G., and Nikaido, H. (2000). High-level fluoroquinolone-resistant clinical isolates of Escherichia coli overproduce multidrug efflux protein AcrA. Antimicrob. Agents Chemother. 44, 3441-3443. doi: 10.1128/AAC.44.12.3441-3443.2000

McClure, R., Balasubramanian, D., Sun, Y., Bobrovskyy, M., Sumby, P., Genco, C. A., et al. (2013). Computational analysis of bacterial RNA-Seq data. Nucleic Acids Res. 41:e140. doi: 10.1093/nar/gkt444

Michel, B. (2005). After 30 years of study, the bacterial SOS response still surprises us. PLoS Biol. 3:e255. doi: 10.1371/journal.pbio.0030255

Mitscher, L. A. (2005). Bacterial topoisomerase inhibitors: quinolone and pyridone antibacterial agents. Chem. Rev. 105, 559-592. doi: 10.1021/cr030 $101 \mathrm{q}$

Nicolas-Chanoine, M.-H., Bertrand, X., and Madec, J.-Y. (2014). Escherichia coli ST131, an intriguing clonal group. Clin. Microbiol. Rev. 27, 543-574. doi: 10.1128/CMR.00125-13

Opperman, T. J., Kwasny, S. M., Kim, H. S., Nguyen, S. T., Houseweart, C., D'souza, S., et al. (2014). Characterization of a novel pyranopyridine inhibitor of the AcrAB efflux pump of Escherichia coli. Antimicrob. Agents Chemother. 58, 722-733. doi: 10.1128/AAC.01866-13

Recacha, E., Machuca, J., Díaz De Alba, P., Ramos-Güelfo, M., Docobo-Pérez, F., Rodriguez-Beltrán, J., et al. (2017). Quinolone resistance reversion by targeting the SOS response. $m$ Bio 8, e00971-17. doi: 10.1128/mBio.00971-17

Robicsek, A., Strahilevitz, J., Jacoby, G. A., Macielag, M., Abbanat, D., Park, C. H., et al. (2006). Fluoroquinolone-modifying enzyme: a new adaptation of 
a common aminoglycoside acetyltransferase. Nat. Med. 12, 83-88. doi: 10.1038/ $\mathrm{nm} 1347$

Rodríguez-Martínez, J. M., Machuca, J., Cano, M. E., Calvo, J., MartínezMartínez, L., and Pascual, A. (2016). Plasmid-mediated quinolone resistance: two decades on. Drug Resist. Updat. 29, 13-29. doi: 10.1016/j.drup.2016. 09.001

Schneider, R., Travers, A., Kutateladze, T., and Muskhelishvili, G. (1999). A DNA architectural protein couples cellular physiology and DNA topology in Escherichia coli. Mol. Microbiol. 34, 953-964. doi: 10.1046/j.1365-2958.1999. 01656.x

Tamae, C., Liu, A., Kim, K., Sitz, D., Hong, J., Becket, E., et al. (2008). Determination of antibiotic hypersensitivity among 4,000 single-geneknockout mutants of Escherichia coli. J. Bacteriol. 190, 5981-5988. doi: 10.1128/ JB.01982-07

Tran, T., Ran, Q., Ostrer, L., and Khodursky, A. (2016). De novo characterization of genes that contribute to high-level ciprofloxacin resistance in Escherichia coli. Antimicrob. Agents Chemother. 60, 6353-6355. doi: 10.1128/AAC.00889-16

Werner, N. L., Hecker, M. T., Sethi, A. K., and Donskey, C. J. (2011). Unnecessary use of fluoroquinolone antibiotics in hospitalized patients. BMC Infect. Dis. 11:187. doi: 10.1186/1471-2334-11-187

White, A. R., Kaye, C., Poupard, J., Pypstra, R., Woodnutt, G., and Wynne, B. (2004). Augmentin (amoxicillin/clavulanate) in the treatment of communityacquired respiratory tract infection: a review of the continuing development of an innovative antimicrobial agent. J. Antimicrob. Chemother. 53(Suppl. 1), i3-i20. doi: 10.1093/jac/dkh050

Yamada, J., Yamasaki, S., Hirakawa, H., Hayashi-Nishino, M., Yamaguchi, A., and Nishino, K. (2010). Impact of the RNA chaperone Hfq on multidrug resistance in Escherichia coli. J. Antimicrob. Chemother. 65, 853-858. doi: 10.1093/jac/ dkq067

Yilmaz, S., Altinkanat-Gelmez, G., Bolelli, K., Guneser-Merdan, D., Ufuk overHasdemir, M., Aki-Yalcin, E., et al. (2015). Binding site feature description of 2-substituted benzothiazoles as potential AcrAB-TolC efflux pump inhibitors in E. coli. SAR QSAR Environ. Res. 26, 853-871. doi: 10.1080/1062936X.2015. 1106581

Conflict of Interest Statement: The authors declare that the research was conducted in the absence of any commercial or financial relationships that could be construed as a potential conflict of interest.

Copyright (c) 2018 Klitgaard, Jana, Guardabassi, Nielsen and Løbner-Olesen. This is an open-access article distributed under the terms of the Creative Commons Attribution License (CC BY). The use, distribution or reproduction in other forums is permitted, provided the original author(s) and the copyright owner(s) are credited and that the original publication in this journal is cited, in accordance with accepted academic practice. No use, distribution or reproduction is permitted which does not comply with these terms. 